\title{
Lumbosacral Plexopathies: Causing by Femoral Head Fracture
}

\author{
Patpiya Sirasaporn
}

\begin{abstract}
Lumbosacral plexopathies is considerably less epidemiologic prevalence. This is due to the relatively protected position of these neural structures. The causes are various such as tumors, hematomas, surgical damage, trauma, diabetes mellitus and paraneoplastic syndrome. The symptoms which are caused by compressing lumbosacral plexus are sensory disturbance and weakness in an affected leg. The author reports a case of 17 -year-old male with history of right femoral head fracture status post open reduction and internal fixation by plate and screw who complained right foot drop and numbness along the leg. His first diagnosis was right sciatic neuropathy. However, 3 months later, he still had difficulty in walking and felt hyperesthesia over lateral aspect of the right leg and the dorsum of foot. His further investigation which was electrodiagnostic study was diagnosed right lumbosacral plexopathies. Although the most common neuropathy after femoral fracture is sciatic neuropathy, lumbosacral plexopathies should be considered.
\end{abstract}

Keywords: Lumbosacral plexopathies; Femoral fracture; Electrodiagnostic study

\section{Introduction}

Lumbosacral plexopathies is considerably less epidemiologic prevalence compared to brachial plexopathies [1]. This is due to the relatively protected position of these neural structures and their decreased accessibility to injury. It may be caused by anatomical abnormalities such as tumors, hematomas, surgical damage and trauma. Moreover, it can be damaged by metabolic insults such as diabetes mellitus, in-

Manuscript accepted for publication May 19, 2014

Department of Physical medicine and Rehabilitation, Faculty of Medicine, Khon Kaen University, Khon Kaen 40002, Thailand.

Email: spatpiya@kku.ac.th

doi: http://dx.doi.org/10.14740/jmc1815w fection and paraneoplastic syndrome. Nevertheless, the most common cause of trauma resulting in lesion affecting the lumbosacral plexus is pelvic fracture [2-4]. The incidence of lumbosacral plexopathies from pelvic fracture is about

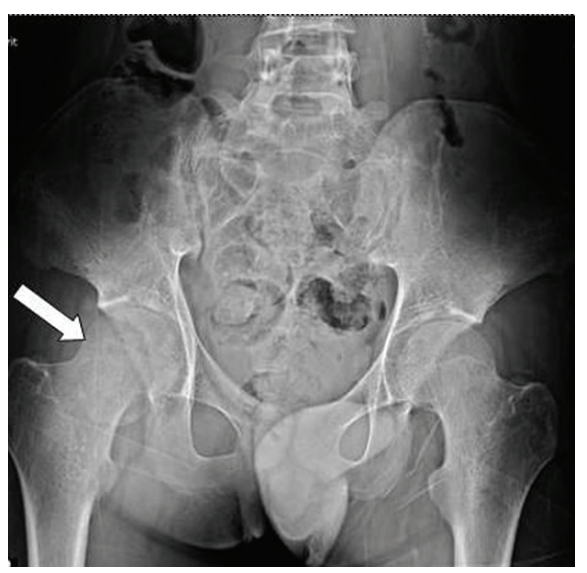

Figure 1. Pelvic radiograph: right femoral head fracture.

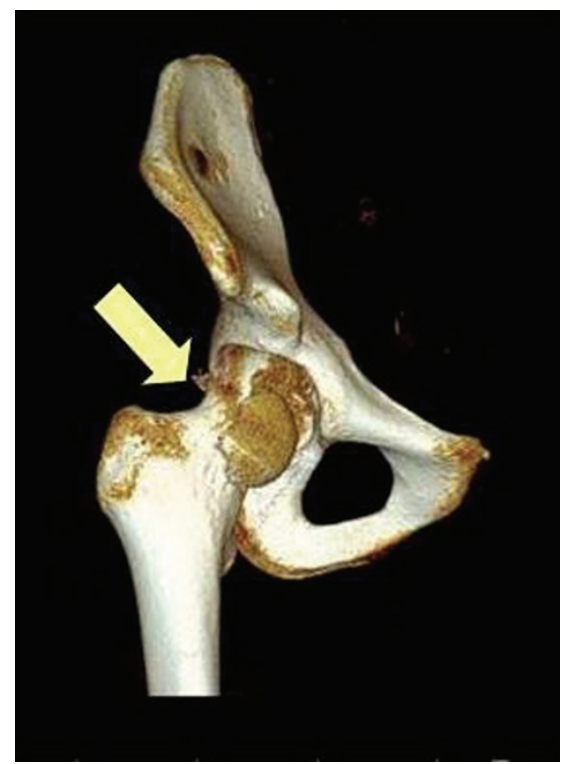

Figure 2. CT scan 3D: Pipkin fracture type II in right hip. 


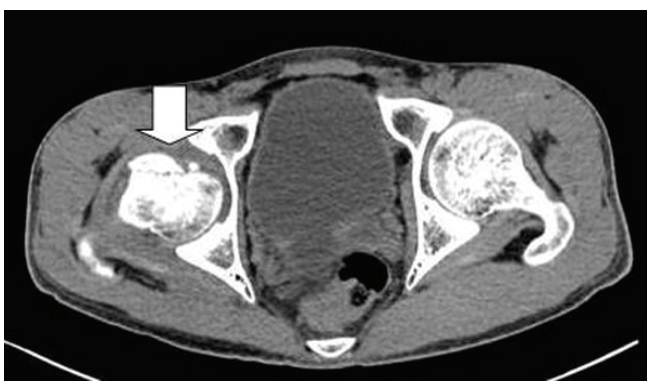

Figure 3. CT scan: hemathrosis in right hip.

9-46\% [3-5] whereas the incidence of lumbosacral plexopathies from femoral fracture is about $0.7 \%$ [6]. The symptoms which are caused by compressing lumbosacral plexus vary according to the structures involved such as sensory disturbance and muscles weakness in an affected leg. Unfortunately, neurological assessment in acute phase is difficult to detect and is generally diagnosed during follow-up.

\section{Case Report}

A 17-year-old male became persistent of right foot drop and numbness along the leg for 3 months. He had history of motorcycle accident. The pelvic radiograph showed fracture of right femoral head (Fig. 1). Computed tomography scan of both hips with 3D reconstruction revealed right femoral head fracture which was proximal to the fovea centralis: Pipkin fracture type II and hemathrosis in the hip (Fig. 2, 3). He underwent open reduction and internal fixation by plate and screw (Fig. 4). He denied any systemic disease. An orthopedic surgeon firstly diagnosed his symptoms as right sciatic neuropathy. However, 3 months postoperatively, his symptoms were not improved and still had paresthesia at right leg and the dorsum of the foot. The electrodiagnostic study was asked to confirm and localize the lesion.

Physical examination revealed muscle atrophy at right leg. Surgical scar at lateral of the thigh was detected. Motor power of right iliopsoas, quadriceps, hamstring, gluteus maximus, gluteus medius were grade 4 all whereas motor power of right tibialis anterior, gastrocnemius were grade 0

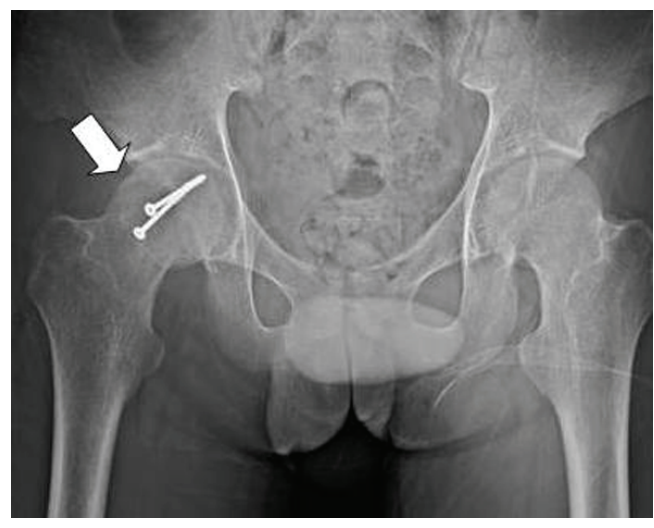

Figure 4. Pelvic radiograph: S/P open reduction and internal fixation by plate and screw in right hip.

all. Impaired pinprick sensation and temperature sensation at right lateral leg and dorsum of the foot were noted. Tinel sign was negative at right popliteal fossa and the fibular head. Deep tendon reflex at right ankle was absent.

Nerve conduction studies (NCS) and needle electromyography were conducted. NCS showed no electrical response at right sensory superficial peroneal and motor peroneal nerves (Table 1). Needle electromyography showed increased insertional activity, variable of spontaneous activity and impairment of recruitment at right biceps femoris (long and short heads), gastrocnemius, gluteus maximus, rectus femoris, tensor fascia latae and tibialis anterior muscles. There were normal insertional activity and no sign of spontaneous activity at right rectus femoris and paravertebral L5 level muscles (Table 2). This result was summarized to right lumbosacral plexopathies. Therefore, ankle foot orthosis which helps to easily walk was ordered to the patient.

Two months later, there was some improvement in right tibialis anterior and gastrocnemius muscles but paresthesia in right leg was unchanged.

\section{Discussion}

Most common neuropathy from femoral fracture is sciatic neuropathy [7]. However, patients with femoral fracture may

Table 1. Sensory and Motor Nerve Conduction Studies

\begin{tabular}{llll}
\hline Nerve & Latency (ms) & Amplitude (mV) & Velocity (m/s) \\
\hline \multirow{2}{*}{ Right sensory superficial peroneal nerve } & NR & NR & NR \\
Right motor peroneal nerve & NR & NR & NR \\
\hline
\end{tabular}

NR: no electrical response. 
Table 2. Needle Electromyography

\begin{tabular}{|c|c|c|c|c|c|}
\hline \multirow{3}{*}{ Muscle } & \multirow{3}{*}{$\begin{array}{l}\text { Insertional } \\
\text { activity }\end{array}$} & \multicolumn{2}{|c|}{ Spontaneous activity } & \multirow{3}{*}{$\begin{array}{l}\text { Amplitude } \\
\text { (mV) }\end{array}$} & \multirow{3}{*}{ Recruitment } \\
\hline & & & & & \\
\hline & & Fibrillation & $\begin{array}{l}\text { Positive sharp } \\
\text { wave }\end{array}$ & & \\
\hline Rt. Biceps femoris (long head) & Increased & $2+$ & $3+$ & $1-3$ & Discrete \\
\hline Rt. Biceps femoris (short head) & Increased & $3+$ & $3+$ & None & None \\
\hline Rt. Gastrocnemius & Increased & $2+$ & $4+$ & None & None \\
\hline Rt. Gluteus maximus & Increased & $1+$ & $2+$ & $1-2$ & Discrete \\
\hline Rt. Tensor fascia latae & Increased & $2+$ & $3+$ & $0.4-1$ & Discrete \\
\hline Rt. Tibialis anterior & Increased & $1+$ & $3+$ & None & None \\
\hline Rt. Rectus femoris & Normal & None & None & $0.5-2$ & Full \\
\hline Rt. Paravertebral (L5 level) & Normal & None & None & - & - \\
\hline
\end{tabular}

have suffered injury to lumbosacral plexus [6]. Patients with lumbosacral plexopathies present frequently with foot drop and numbness or paresthesia in the lateral leg and the dorsum of foot which should be differentiated from sciatic neuropathy. Neurological findings of lumbosacral plexopathies include not only weakness of ankle dorsiflexion and plantar flexion but there also is variable weakness of the gluteus and hamstring muscles. The hallmarks of electrodiagnostic study for lumbosacral plexopathies are spontaneous activity in peroneal, tibial, sciatic, superior gluteal and inferior gluteal innervated muscles. The motor and sensory nerve conduction studies of an affected lower limb reveal diminished or no electrical response [8].

This report showed lumbosacral plexopathies from uncommon cause: femoral fracture. The causes of lumbosacral plexopathies in this patient may be a result of high speed deceleration injuries sustained in vehicle accidents. Due to the relatively clinical presentations of lumbosacral plexopathies and peripheral nerve lesion, electrodiagnostic study is an important key for confirm diagnosis.

\section{Conclusion}

Diagnosis of lumbosacral plexopathies is difficult to differentiate between a lumbosacral plexus and peripheral nerve lesion. When careful neurological examination is combined with an electrodiagnostic study, a number of lumbosacral plexopathies may be increased. Therefore, clinicians should carefully assess femoral fracture patients who have sensory disturbance and weakness in an affected leg not only for sciatic neuropathy but also lumbosacral plexopathies.

\section{Grant Support}

No grant support, no commercial party having a direct financial interest in the results of the research supporting this article has or will confer a benefit upon the authors or upon any organization with which the authors are associated.

\section{References}

1. Goodell CL. Neurological deficits associated with pelvic fractures. J Neurosurg. 1966;24(5):837-842.

2. Sugimoto $\mathrm{Y}$, Ito $\mathrm{Y}$, Tomioka $\mathrm{M}$, Tanaka $\mathrm{M}$, Hasegawa Y, Nakago K, Yagata Y. Risk factors for lumbosacral plexus palsy related to pelvic fracture. Spine (Phila Pa 1976). 2010;35(9):963-966.

3. Jang DH, Byun SH, Jeon JY, Lee SJ. The relationship between lumbosacral plexopathy and pelvic fractures. Am J Phys Med Rehabil. 2011;90(9):707-712.

4. Chiodo A. Neurologic injury associated with pelvic trauma: radiology and electrodiagnosis evaluation and their relationships to pain and gait outcome. Arch Phys Med Rehabil. 2007;88(9):1171-1176.

5. Goldberg G, Goldstein H. AAEM case report 32: nerve 
injury associated with hip arthroplasty. Muscle Nerve. 1998;21(4):519-527.

6. Kutsy RL, Robinson LR, Routt ML, Jr. Lumbosacral plexopathy in pelvic trauma. Muscle Nerve. 2000;23(11):1757-1760.
7. Yuen EC, Olney RK, So YT. Sciatic neuropathy: clinical and prognostic features in 73 patients. Neurology. 1994;44(9):1669-1674.

8. Strakowski JA. Electrodiagnosis of plexopathy. PM R. 2013;5(5 Suppl):S50-55. 